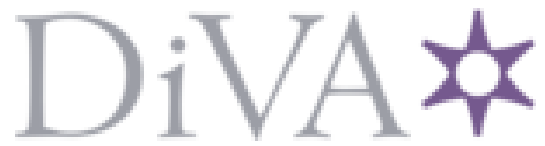

http://www.diva-portal.org

Postprint

This is the accepted version of a paper presented at 1st IEEE Conference on Network Softwarization (NetSoft), 13-17 April 2015, London.

Citation for the original published paper:

Vestin, J., Kassler, A. (2015)

QoS Enabled WiFi MAC Layer Processing as an Example of a NFV Service.

In: Network Softwarization (NetSoft), 2015 1st IEEE Conference on (pp. 1-9). IEEE conference proceedings

https://doi.org/10.1109/NETSOFT.2015.7116164

N.B. When citing this work, cite the original published paper.

Permanent link to this version:

http://urn.kb.se/resolve?urn=urn:nbn:se:kau:diva-38735 


\title{
QoS Enabled WiFi MAC Layer Processing as an Example of a NFV Service
}

\author{
Jonathan Vestin and Andreas Kassler \\ Computer Science Department \\ Karlstad University \\ Karlstad, Sweden \\ Email: \{jonathan.vestin, andreas.kassler\}@kau.se
}

\begin{abstract}
The Configuration and Management of large WLAN deployments is a challenge and available tools to ease such deployments and introduce new services are either commercial or very inflexible. In this paper, we present a different approach to such challenges called QoS enabled CloudMAC, which is to the best of our knowledge the first step towards QoS enabled WiFi MAC layer processing as an example of Network Function Virtualization. By moving the MAC layer processing to the cloud and integrating our architecture with QoS aware OpenFlow deployment, a software defined networking approach, we achieve a new level of flexibility, control and reconfigurability. CloudMAC Access Points (AP) just forward MAC layer frames towards a set of VMs (Virtual Access Points - VAP) that are responsible for processing MAC layer data and management frames (such as beacons, probe requests, etc). We have extended the SDN that connects the VAPs with the physical APs to support different packet prioritisation strategies such as HTB, SFQ, or FQ_CoDel. Our SDN controller is based on OpenDaylight which creates layer 2 forwarding rules that effectively prioritise CloudMAC traffic over legacy traffic. Our evaluation in a real testbed shows that packet prioritization strategies, especially FQ_CoDel, lead to good throughput and low latency for CloudMAC traffic while at the same time maintaining low latency for background traffic.
\end{abstract}

\section{INTRODUCTION}

The world today is connected more than ever before and mobile and wireless devices have seen significant growth during the last years. Large WiFi networks, such as enterprise WLANs or WLANs deployed by network operators introduce new difficulties concerning configuration and connectivity. Users expect constant seamless mobility, while real-time handover support for large WiFi networks is difficult to achieve due to the way WLAN networks are designed. In order to cope with the ever increasing number of devices and Access Points (AP), new management approaches are required that allow to introduce novel services much more rapidly and flexible.

In the fixed network, Software Defined Networking (SDN), based on e.g. OpenFlow [1] has demonstrated to be an important technology driver for flexible service development. In recent years, SDN has rapidly transformed campus networks, data centres, and the cloud in order to provide a more flexible network architecture based on its simple per flow management. By programming SDN controller [1] applications using e.g. Java or Python, important use cases such as load-balancing or QoS management can be rapidly deployed without manual configuration. Enabling OpenFlow for WLAN presents an interesting perspective towards more manageable, agile large WLAN deployments that can scale with network demand. For example, using SDN to monitor network traffic flows, policies could be rapidly installed that specify how the traffic should be routed, which application flows should be prioritised or what APs should be powered down to save energy.

NFV (Network Function Virtualization) [2] has been recently introduced due to the possibility to virtualize network functions, such as gateways, firewalls, Network Address Translation (NAT), or Domain Name Service (DNS). The key idea is to replace expensive customised hardware (such as a dedicated firewall box) with virtual functions that can be implemented inside e.g. Virtual Machines and deployed inside e.g. a Cloud based on OpenStack [3]. NFV allows rapid and dynamic deployment of new features into a network, providing more agile deployment and management. In addition, it scales easily with demand by adding new instances (e.g. deploy new VMs) of such service or add more processing power on demand (e.g. by adding more virtual CPUs). An interesting example of NFV in the area of WLAN is CloudMAC [4], which virtualises 802.11 MAC frame processing, separating the physical AP from the logical one denoted as VAP (Virtual Access Point). The VAP is hosted inside a VM in the co-located Data Centre and processes all MAC layer management frames. As a result, association state is stored in the cloud, and the user is always associated with its dedicated AP, no matter what physical AP the WiFi packets pass through. By using a SDN based network that connects the physical APs with the VAP, handovers can be made seamless by changing forwarding rules using a CloudMAC SDN control application. Furthermore, the configuration of multiple APs are stored in the same VM, making configuration vendor independent and simple. As such, CloudMAC does not require any change to the mobile node itself. However, when operating CloudMAC traffic over a network, CloudMAC traffic needs to share resources with background traffic. If the network is congested, latency for CloudMAC traffic will increase. As a result, important WiFi frames such as Probe Response might be delayed or lost, severely penalising performance of CloudMAC.

The key contribution of this paper is the design and implementation of QoS management for CloudMAC. First, we extend current OpenFlow infrastructure to support flexible traffic prioritisation and several queue management strategies 
such as HTB, SFQ, or FQ_CoDel on Open vSwitch enabled routers and switches. Second, we modify CloudMAC to utilize such prioritisation strategies by developing an OpenDaylight application that installs dedicated queuing strategies on those modified OpenvSwitches using a high performance kernel based forwarding data path. Finally, we develop forwarding rules that prioritise CloudMAC traffic over legacy traffic. An evaluation in our testbed shows that using those queuing strategies under heavy cross traffic and congested links indeed effectively prioritise CloudMAC traffic and lead to higher connection success rate, better throughput and lower latency for CloudMAC traffic while at the same time maintaining low latency for the background traffic.

The remainder of this paper is structured as follows. Chapter II presents related work and how they compare to our solution. In Chapter III, we discuss the design of CloudMAC and how we extend our architecture to support flexible traffic prioritisation and different queuing strategies. Chapter IV evaluates CloudMAC performance using various queueing strategies in a real testbed. Finally, Chapter V concludes the paper and presents ideas for future work.

\section{RELATED WORK}

There have been several developments in providing a vendor independent configuration of APs, such as CAPWAP [5], Odin [6] and CloudMAC [4]. In CAPWAP, the frame generation is split between the controller and the WTPs, providing centralised AP configuration, authentication and association. Odin works by using LVAPs (Light Virtual Access Points) running at each AP. In odin, each MN connecting to the network is assigned a new LVAP and an unique BSSID. The APs also run a single Agent managing the LVAPs which informs the Master of the network state. The Master in turn can perform a handover by moving a LVAP from one AP to another. This also provides vendor independent configuration. Odin does not support any specific QoS configuration. CloudMAC on the other hand performs all 802.11 MAC layer processing, except wireless ACK frames, in the VAP. The advantage of CloudMAC as opposed to systems like CAPWAP or Odin is that almost all processing is done at the VAP and that the implementation is very simple. This allows the WTPs to be lightweight devices, only required to forward frames and generate an wireless ACK for each received frame. All other services can be implemented by OpenFlow controllers or within VMs in a co-located cloud. With the introduction of QoS management for CloudMAC, we can now effectively prioritize CloudMAC traffic over other traffic leading to improved performance.

Recently, there has been done some research of how to integrate QoS management into SDN enabled networks. QoSFlow [7] is one such development, that adds extensions for QoS configuration to an OpenFlow enabled software switch. This allows for easy configuration of QoS strategies using OpenFlow messages. However, this approach suffers from a lack of performance due to the software switch used (54 Mbps, compared to $800 \mathrm{Mbps}$ without the software switch). Our approach uses a similar design but instead uses Open vSwitch, a software switch with a kernel datapath providing much higher performance. In fact, with our approach we can sustain more than $500 \mathrm{Mbps}$ on the same devices as authors from [7] use. In addition, we have determined how such improved performance can be applied effectively for QoS enabled NFV for WiFi MAC layer processing in the cloud. FlowQoS [8] uses Open vSwitch to provide QoS in home networks using consumer grade hardware. FlowQoS focuses on flow classification and uses the HTB QoS strategy to prioritise traffic automatically (or per user-defined rules). However, flow classification is done within a SDN controller that runs on small devices severely limiting performance. Our solution targets different use cases, such as the prioritisation of WiFi MAC layer frames in SDN enabled switches to support NFV.

HTB [9] is a commonly used queueing strategy, which allows classifying different types of traffic into queues. Each queue can be set up with a rate limit and priority. Due to its strict prioritization, HTB can achieve high throughput and low latency for queues with high priority. However, HTB can be difficult to configure in large networks, because each queue needs to be separately configured. SFQ [10] provides queue separations of flows without the need for classification. It uses a fair queueing algorithm, that assigns each data flow a queue using a hashing algorithm and uses a roundrobin scheduling dequeues on all queues. The hashing method could assign two flows to the same queue, therefore the hashing algorithm is perturbed with a configurable interval. CoDel [11] can be seen as a successor to RED [12], which when the queue delay of a packet reaches a configured interval, packet drop probability increases until the queue delay is lowered below the interval. FQ_CoDel [13] separates the flows into multiple subqueues and uses a modified Deficit Round Robin scheduling algorithm. CoDel is applied to each of the subqueues in FQ_CoDel and the dropping is adjusted in order to keep the queue inflicted latency within delay bounds that can be specified.

\section{QOS FOR CLOUdMAC}

\section{A. Design}

CloudMAC [4] can be seen as a so called Split MAC approach for 802.11 based WLANs which allows offloading of 802.11 MAC frame processing to one or more mac-processor machines, which typically are placed in a co-located data centre. CloudMAC separates the AP into two entities, denoted as VAP (Virtual Access Point) and WTP (Wireless Termination Point). The WTP is a stripped down WiFi access point, which forwards all 802.11 MAC frames to the VAP. CloudMAC assumes a SDN infrastructure like OpenFlow enabled switches to transport the MAC frames between the VAP and the WTP. An OpenFlow control application establishes the binding between the VAP and WTP and establishes proper forwarding paths. As the VAP processing is based on a software driver, it is possible to virtualise the wireless MAC frame processing and deploy it within a virtual machine within e.g. a private 
cloud like OpenStack. This allows to create multiple instances of VAPs that process MAC frames, which allows to scale with increasing load in the network, for example. As such, CloudMAC is an example of NFV in relation to WiFi MAC frame processing. This has several advantages to regular wireless frame processing including increased processing power, seamless handover from one AP to another one, dynamic power on/off of access points and a unified configuration interface, as we will show below.

\begin{tabular}{l} 
VAP $\rightarrow$ WTP Header \\
\begin{tabular}{|c|c|c|}
\hline \multicolumn{3}{|c|}{ Destination Address } \\
\hline Signal & Rate & Source VAP ID \\
\hline Ethertype & Radiotap Header.. \\
\hline
\end{tabular} \\
\hline
\end{tabular}

WTP $\rightarrow$ VAP Header
\begin{tabular}{|c|c|}
\hline \multicolumn{2}{|c|}{ Destination Address } \\
\hline \multicolumn{2}{|c|}{ Source Address } \\
\hline Ethertype & Radiotap Header.. \\
\hline
\end{tabular}

Fig. 1: CloudMAC header

Before the WTP sends the frame to the VAP, it adds an additional CloudMAC header (see Figure 1) to the packet. This header looks just like a standard ethernet header, however it carries a special ethertype signifying that it is a CloudMAC packet. This requires the switch to be able to recognize and process CloudMAC packets separately from normal packets. We achieve this through using an OpenFlow-enabled network, where rules match the special CloudMAC ethertype. This allows the packet to be processed by normal OpenFlow rules with existing controllers. When the packet from the WTP arrives at the VAP, the header is removed and the packet is processed normally in software through a modified version of the mac80211_hwsim driver (which provides a virtual interface that applications like hostapd can use). When packets are sent to this interface (in the reverse direction, from VAP to WTP), they are encapsulated again in a CloudMAC header at the VAP. The CloudMAC header has two additional fields, transmission power and rate, which are set by the mac80211_hwsim driver. When the WTP receives the packet, the CloudMAC header is removed and the packet is transmitted to the WLAN.

When a MN connects first to the CloudMAC enabled network, the CloudMAC application at the OpenFlow controller (in our new version, we use OpenDaylight [14] due to it being one of the most commonly used OpenFlow controllers available) establishes the binding between the VAP and the WTP having received the packet with the strongest signal strength. All WTPs run their WiFi cards in monitoring mode and transparently forward received MAC layer frames to the first OpenFlow enabled switch. The CloudMAC application on the controller then creates rules to forward just a single copy of such MAC layer frames towards the VAP, all MAC layer frames received from other WTPs will be dropped at the first switch. In addition, we have implemented a control application on the WTP that enables the generation of 802.11 Wifi ACK frames from the proper WTP, because we were not able to generate the ACKs fast enough in software from the VAP to fulfil the 802.11 MAC layer timing requirements.

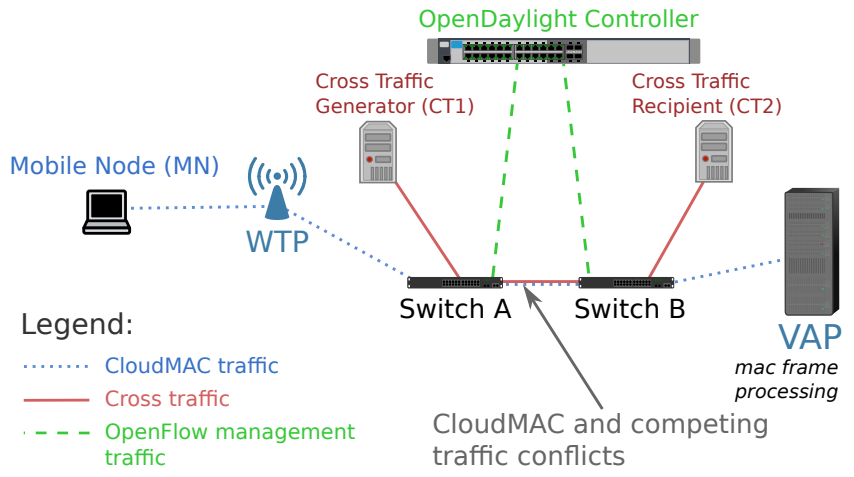

Fig. 2: CloudMAC network overview and testbed setup

As all WTPs send each WiFi MAC frame to the OpenFlow enabled switch, and the switch only forwards the packets from the WTP which has the highest signal strength, the controller can automatically perform a seamless wireless handover. In order to do so, the controller periodically changes flow rules to forward all packets from all WTPs to the controller application. The controller then examines, which packet from what WTP for a given MN has the highest RSSI and modifies flow rules accordingly, and returns packet back to the switch. For example, let us assume that a MN is using WTP A (the flow rules forward just packets from WTP A towards the VAP and only WTP A sends MAC layer ACKs to the MN), but enters transmission range of WTP B. After the next monitoring period, the OpenDaylight controller detects that MAC frames now arrive at WTP B with higher SNR. The controller then sends a command to WTP A to disable MAC layer ACKs for this $\mathrm{MN}$ and alters flow rules to drop packets coming from this WTP for the given MN. At the same time, it instructs WTP B to send ACKs to MN and creates a forwarding path for the MN through WTP B to the VAP (i.e. MN $\leftrightarrow$ WTP A $\leftrightarrow$ OF Network $\leftrightarrow$ VAP changes to MN $\leftrightarrow$ WTP B $\leftrightarrow$ OF Network $\leftrightarrow$ VAP). As association states are kept in the VAP, we do not require reassociation and such handovers can be implemented seamlessly [4]. The smaller the monitoring period, the faster HOs can be implemented but the higher the processing overhead at the controller.

In addition, the OpenDaylight controller can automatically detect and configure new WTPs and VAPs if they are added to the network. The VAPs are detected when broadcasted beacons (generated at the VAP) reach the OpenDaylight controller. The WTPs are detected by a special packet they send at a configurable interval. This packet contains information about 
the WTP, such as the IP address. This allows for dynamically powering on/off and adding/removing VAPs and WTPs depending on the utilisation of the network. Because we have reserved two fields in the CloudMAC header to encode the rate and transmission power, an application at the Controller can easily change the transmission power and rate used by the WTP. This application uses the mod_dl_src action to rewrite the values of the txpower and rate fields in the CloudMAC header on a per-flow basis. When the WTP receives CloudMAC packets, it removes the header and uses the rate and txpower as encoded in the header to send the packets on the $\mathrm{WiFi}$ interface.

\section{B. QoS for CloudMAC}

CloudMAC traffic may coexist with normal traffic (e.g. heavy TCP based downloads) within the SDN enabled network that may span e.g. a large enterprise or campus area. This may lead to high latency and packet loss for CloudMAC traffic. Through initial experiments using CloudMAC, we have seen that several CloudMAC packets may get lost or delayed if CloudMAC traffic is transmitted over a shared network. As some of the packets are time sensitive (such as wireless management frames), this may result in performance loss and connection instability for the CloudMAC traffic in a CloudMAC enabled network. As we will demonstrate later, tests have shown that the probability of successfully establishing a connection may be significantly reduced (from $98 \%$ to $55 \%$ ) when cross traffic congests the network and CloudMAC traffic is delayed or lost.

We have implemented a SDN based approach to prioritize CloudMAC traffic, because CloudMAC assumes a SDN enabled network to provide connectivity between VAP and WTP. In our approach, we assume that SDN enabled switches run Open vSwitch and we have extended Open vSwitch with flexible queue management and configuration strategies, including SFQ [10], CoDel [11] and FQ_CoDel [11]. We connected the switches running Open vSwitch to an SDN controller and implemented, in our CloudMAC SDN application, a set of rules, that map CloudMAC traffic to dedicated queues which have been configured in order to flexibly prioritize CloudMAC traffic. To configure QoS for Open vSwitch, the ovs-vsct 1 tool is used. This tool modifies the ovsdb database, which is monitored by ovs-vswitchd user-space daemon. The user-space daemon then updates the linux qdisc configuration. The OpenDaylight SDN controller has an ovsdb interface, which can be used to configure the ovsdb instance running on the Open vSwitch enabled software switches. Alternatively, we could use hardware switches that support the ovsdb protocol. As new CloudMAC flows are added, the switches which the flow passes through are configured with a QoS strategy. In case of the classless strategies like SFQ, CoDel and FQ_CoDel, no queue configuration is required. However, with classful strategies like HTB, each switch is also configured with two different queues, one with higher priority than the other. OpenDaylight is then used together with the enqueue action to enqueue the CloudMAC traffic in the high priority queue using OpenFlow v1.3 OFPAT_SET_QUEUE.

In the following we provide some example code that con-

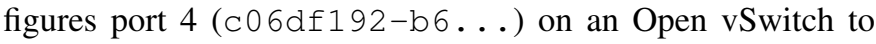
prioritize CloudMAC traffic using HTB using OpenDaylights RESTCONF API:

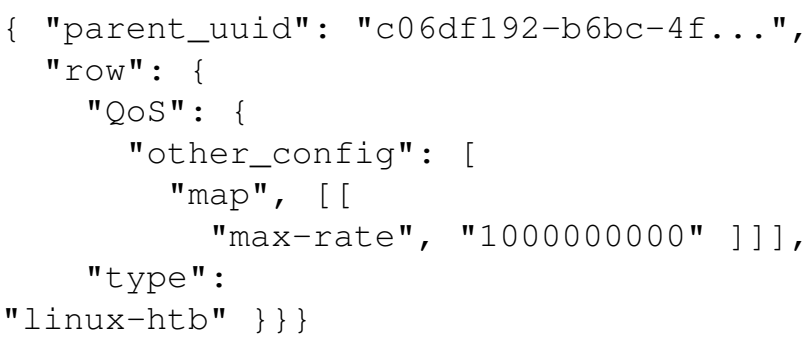

Configuring the CloudMAC Queue connected to QoS uuid 109b55a6-1e...:

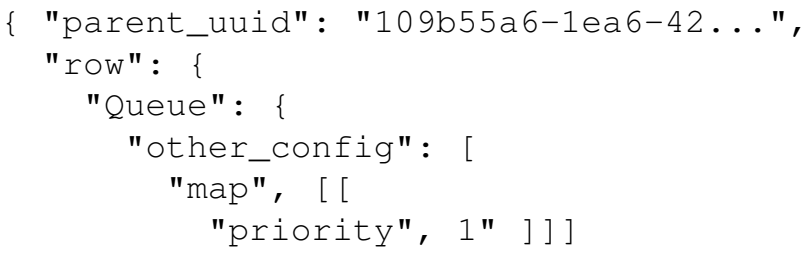

\} \} \}

Using the (simplified) flow table:

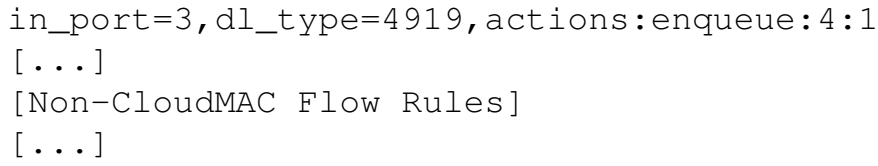

\section{Implementation}

In Open vSwitch, the available QoS strategies are listed in the tc_ops struct in the list tcs. When a QoS strategy is configured using ovsdb, a search through the tcs list is performed, where it checks if the field ovs_name matches the type configued in the qos table in ovsdb. The tc_ops struct has pointers to functions for setting up that particular QoS strategy.

We extended Open vSwitch to support SFQ, CoDel and FQ_CoDel. This was done by extending tcs to include tc_ops_sfq, tc_ops_codel and tc_ops_fqcodel. For each of these structs, we implemented the required functions to allow for qdisc creation and deletion:

tc_install Takes a net device (netdev) and a key-value map (smap) as parameters. The key-value map contains the information about the QoS configuration parameters from the ovsdb database. This function should set up the net device with the parameters specified in the database (provided through smap). There is no need to delete the previous qdisc, as this is managed by Open vSwitch. If this option is null, the qdisc cannot be installed (such is the case for the default qdisc tc_ops_other).

tc_destroy Destroys the data structures which is part of the tc struct. 
qdisc_get Retrieves the configuration of the qdisc and returns it to the caller. The parameters provided are the net device, and a key-value map which the function should fill with appropriate key-value pairs.

qdisc_set Reconfigures the qdisc bound to the provided net device parameter. The net device should be configured according to the key-value map provided as a parameter.

class_get Retrieves the configuration of the queues and returns it to the caller. The parameters provided are the net device, and a key-value map which the function should fill with appropriate key-value pairs.

class_set Reconfigures the queue which is provided as a parameters. Should be reconfigured according to the keyvalue map also provided as a parameter.

class_delete Deletes the queue from the tc struct.

The changes made to Open vSwitch are available at: http: //enterprise.cse.kau.se/ jona_vest/p/ovs/

\section{Evaluation}

In this section, we evaluate the impact of cross traffic on the performance of CloudMAC and test, how different queueing strategies can effectively improve CloudMAC performance by reducing latency and throughput without impacting background traffic too much. We will first evaluate our Open vSwitch extensions and then focus on CloudMAC performance.

\section{A. Forwarding Performance of Open vSwitch kernel data path extensions}

In order to evaluate the performance of our Open vSwitch QoS extensions we set up a testbed consisting of two machines for traffic generation connected to two ports of a single TPLink TL-WR1034ND router. TP-link is running OpenWrt 12.09 and Open vSwitch 2.0 with our QoS extensions installed, providing support for OpenFlow 1.3 and a kernellevel datapath provided by Open vSwitch. Figure 3 shows the TCP performance of a single TCP upload flow from the traffic generator machine to the traffic receiver machine using the implemented queueing strategies. We used 15 Repetitions, each one with a single $60 \mathrm{sec}$ TCP Stream using netperf-wrapper. The reference setup is without using Open vSwitch, but rather built-in hardware switching using the TP-link switch fabric. No TC denotes the setup where we use Open vSwitch on the TP-link device without any queueing strategy configured.

As we can see from Figure 3, the performance loss of using the Open vSwitch software switch, compared to the hardware switching is around $332 \mathrm{Mbps}$. This is due to that all switching is done in software, instead of in hardware. However, 534.02 Mbps is still high compared to other approaches such as QoSFlow [7], which uses a user level datapath. We can see a significant loss in TCP throughput using the HTB queuing strategy, which is due to the increased processing requirement of HTB. SFQ, CoDel and FQ_CoDel however provide reasonably high performance. During this test, the CPU utilization of the switch was $100 \%$, which is the reason why the software switch performs worse than the

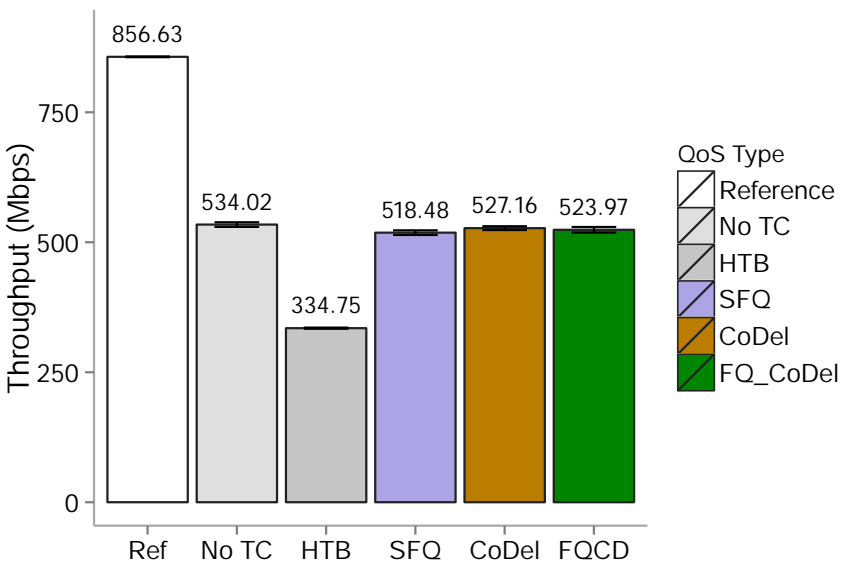

Fig. 3: TCP Performance of implemented queuing strategies (TP-Link TL-WR1034ND)

hardware switch. Clearly, the CPU of the TP-Link switch is the bottleneck. In order to not bias our results further, in all following tests, we use software rate-limiting to $100 \mathrm{mbps}$. However, we can conclude that our approach, which combines OpenWRT with several queuing strategies using Open vSwitch is among the best performing solutions that provide OpenFlow v1.3 capabilities for consumer grade devices.

\section{B. QoS for CloudMAC - testbed and evaluation scenarios}

For testing the performance of CloudMAC QoS extensions, we have set up a testbed which consists of one WTP, one VAP, two software switches running Open vSwitch, two machines for cross-traffic generation (CT1 \& CT2) and one WiFi mobile node (MN). We used a Cambria GW2358-4 device as WTP, equipped with an Atheros based (AR5212) WLM54AG wireless card running OpenWrt 12.09 (Attitude Adjustment) with a CloudMAC version ported to OpenWrt 12.09. We used Dell Optiplex 755 desktop machines running Ubuntu 13.10 (Saucy Salamander) for VAP and cross-traffic generators. The cross traffic machines were running the Linux kernel version 3.11.024-generic and the VAP was running the Linux kernel version 2.6.32-22-generic. We used TP-Link TL-WR1034ND routers for OpenFlow enabled software switches, running OpenWrt 12.09 and Open vSwitch 2.0 with our QoS extensions installed. This provides support for OpenFlow 1.3 and a kernel level datapath. We used a ASUS Eee PC 1215 equipped with an Atheros (AR9271) based TP-Link TL-WN722NC as mobile node running Ubuntu 14.04 LTS (Trusty Tahr) (Linux kernel version 3.13.0-30-generic).

Figure 2 shows our topology for evaluating the impact of crosstraffic (CT1 $\leftrightarrow$ CT2) on CloudMAC traffic (VAP $\leftrightarrow \mathrm{MN}$ ) over the bottleneck (Switch A $\leftrightarrow$ Switch B). The Switch A $\leftrightarrow$ Switch B link is rate limited to $100 \mathrm{Mbps}$ using TBF (Token Bucket Filter) software rate limiting with a $100 \mathrm{~ms}$ long queue. Seven different configurations were tested: without queuing strategy (using plain FIFO instead) on the OpenFlow enabled switches and no cross traffic, no special queuing strategy but 
with cross traffic, using HTB with cross traffic (CloudMAC: priority $=1$, cross traffic: priority $=99$ ), CoDel with cross traffic and FQ_CoDel with cross traffic. Two different reference setups were used. The first setup (Reference 1) is a normal BSS, where the station exchanges traffic with the AP. Here, traffic is exchanged between the STA and the AP. In the second reference setup (Reference 2), the station is connected to the AP, which then routes the traffic through our two TPlink switches to a server, emulating a CloudMAC wireless network. The throughput and RTT (round-trip time) tests were performed using a tool called netperf-wrapper [15]. To generate the cross traffic, netperf-wrapper's realtime response under load (rrul) test was used, which creates four bidirectional TCP flow. The throughput was measured using the tcp_1up_noping test and round trip time was measured using the ping test. The WiFi connection success rate was tested by repeatedly disconnecting and connecting to the network using a custom made script.

\section{Impact of cross traffic and queueing strategies on latency and connection success rate for CloudMAC}

Figure 4 illustrates, how different queueing strategies affect the connection success probability of CloudMAC. We used a Broadcom BCM 4313 wireless network card at the mobile node which made 1500 connection attempts using various queueing strategies. Between each connection attempt, the interface was removed, the driver removed and inserted again, a new interface was created and a connection attempt was made. This was to ensure that each connection attempt would behave like a new connection to the network.

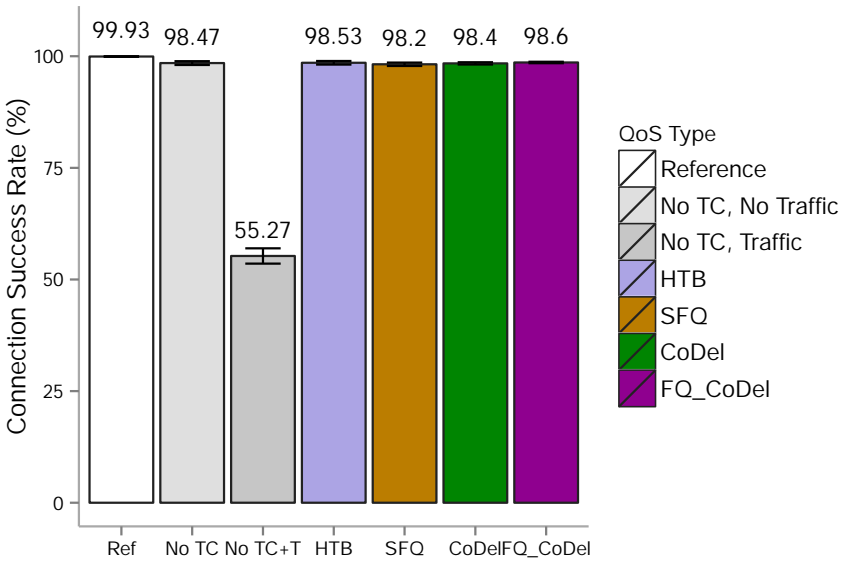

Fig. 4: Connection success rate using Broadcom WiFi card

We can see from Figure 4 that the connection success probability of CloudMAC (without traffic control and crosstraffic - No TC, No Traffic) is slightly worse than the reference network, because of the increased number of hops between VAP and WTP, causing a higher RTT. However, with crosstraffic (No TC, Traffic), the CloudMAC network connection probability decreases to $55 \%$. This is because when cross traffic saturates the link, latency for latency sensitive MAC frames such as wireless management frames increases. When such frames are lost or come too late, the MN will attempt to resend them, eventually give up connecting. As a consequence, the decrease in connection success probability can be directly related to the increased latency and packet loss due to cross traffic. The broadcom drivers specify a $50 \mathrm{~ms}-150 \mathrm{~ms}$ delay tolerance for probe responses. If this timeout is reached, the connection attempt fails. However, other network cards have different delays. We have observed that for example Intel WiFi cards have around $10 \mathrm{~ms}$ delay tolerance, while Atherosbased cards have up to $1000 \mathrm{~ms}$. In addition, we can see that all queueing strategies that we tested (HTB, SFQ, CoDel, FQ_CoDel) provide good connection success probabilities. This is because all the tested strategies provide CloudMAC traffic with a low enough RTT and packet loss. The RTT for each of the queueing strategies is later examined in detail in Figure 6.

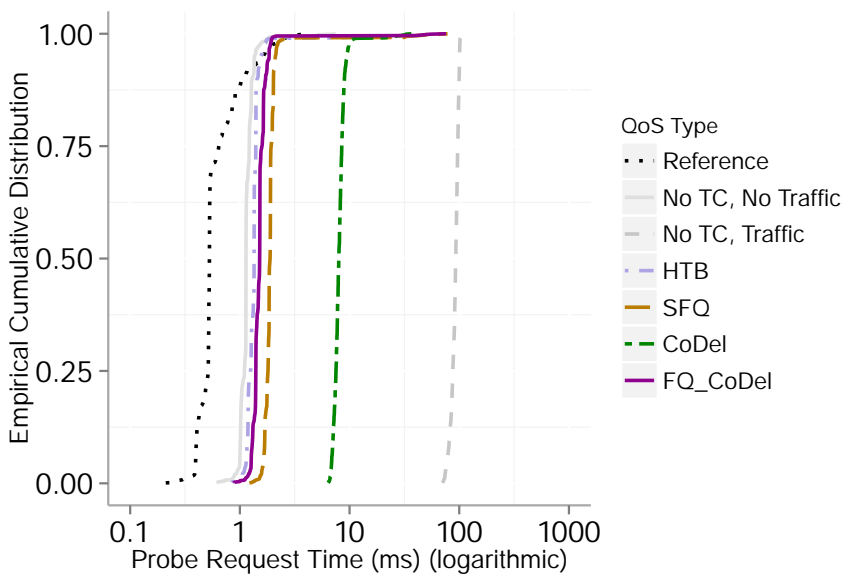

Fig. 5: Probe response time using Atheros WiFi card

Figure 5 shows the ECDF of the probe response time, which is the time difference between the probe request was sent from the $\mathrm{MN}$ and the probe response has been received at the MN. To calculate probe response time, we implemented a small tool that listens to a monitor interface while generating a probe request and waits for the probe response. In CloudMAC, Probe Request and Response are exchanged between $\mathrm{MN}$ and VAP through the WTP over the SDN enabled network. In contrast, Reference 1 in the figure shows the probe response time of a traditional unloaded WLAN, where messages are just exchanged over the air interface. As the network is not loaded, $85 \%$ of the probe response time is under $1 \mathrm{~ms}$. However, when using CloudMAC over an unloaded network, Probe Response Time is slightly higher due to the additional hops and header processing at the VAP and WTP. For example, while in traditional WLAN, 50\% of Probe Response Time is below $0.53 \mathrm{~ms}$, in CloudMAC more than $50 \%$ have a Response Time of $1.14 \mathrm{~ms}$. When the network is loaded, Probe Response Time increases to around $93 \mathrm{~ms}$. For example, 50\% of Probe Response Time under Load is more than $92.9 \mathrm{~ms}$. The impact of different prioritisation strategies is very noticeable. 
For example, when the network is loaded and we are using FQ_CoDel, 95\% of the Probe Response Time is under $1.8 \mathrm{~ms}$, while for CoDel, the Response Time goes up to $9.4 \mathrm{~ms}$ for more than $95 \%$ of the Probe Responses which shows the effectiveness of FQ_CoDel.

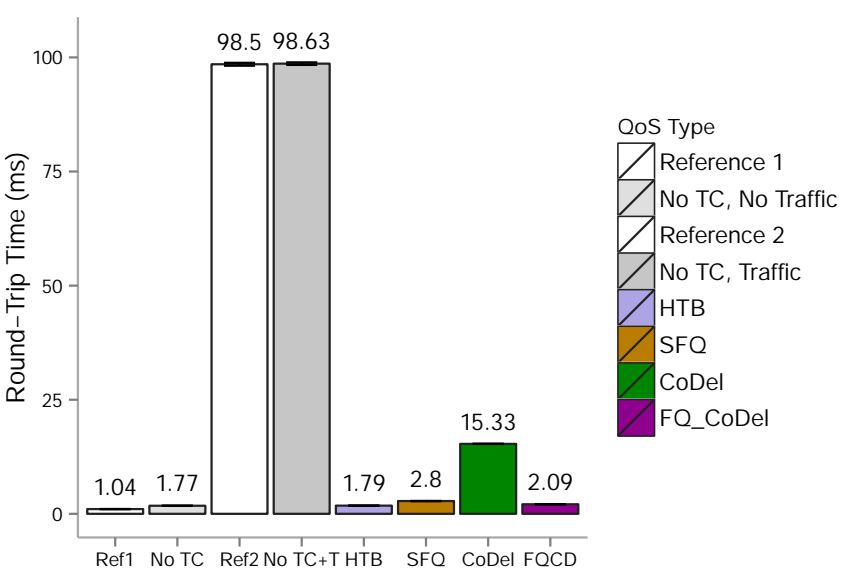

Fig. 6: RTT between $\mathrm{MN}$ and AP/VAP

Figure 6 shows the measured RTT for CloudMAC traffic between the MN and the VAP (data path: MN $\leftrightarrow$ WTP $\leftrightarrow$ A $\leftrightarrow \mathrm{B} \leftrightarrow \mathrm{VAP}$ ) using different queueing strategies. We used 15 repetitions, each running for 60 seconds and the figure shows the mean RTT and the standard deviation. As we can see (Fig. 6), the RTT of CloudMAC traffic between the VAP and the $\mathrm{MN}$ is around $1.77 \mathrm{~ms}$. This is $0.729 \mathrm{~ms}$ longer compared to a traditional wireless network where the $\mathrm{MN}$ is directly connected to the AP (Reference 1: $1.041 \mathrm{~ms}$ ). We can also see that when the $\mathrm{A} \leftrightarrow \mathrm{B}$ link is congested, the average RTT of the CloudMAC traffic between the MN and the VAP is increased to $98.632 \mathrm{~ms}$ (No TC, Traffic: $98.632 \mathrm{~ms}$ ). This is $0.134 \mathrm{~ms}$ longer than a traditional routed WLAN network with cross traffic (Reference 2: $98.498 \mathrm{~ms}$ ). The increase in RTT is caused by the additional VAP processing and the CloudMAC header. In addition, from the figure we see that queueing strategies on the congested link $\mathrm{A} \leftrightarrow \mathrm{B}$ have significant impact on the RTT. For example, CoDel reduces the RTT in a congested network from $98.632 \mathrm{~ms}$ to $15.325 \mathrm{~ms}$, because all flows are processed in a single queue within CoDeL. The fair-queueing traffic control provides an even greater RTT reduction between the MN and the VAP (SFQ: $2.8 \mathrm{~ms}$ and FQ_CoDel: $2.094 \mathrm{~ms}$ ) because CloudMAC and background flows are separated into individual queues. HTB provides the best RTT reduction of the tested queueing strategies at $1.791 \mathrm{~ms}$. This is due to the strict prioritisation of CloudMAC traffic over cross traffic which comes at the expense of much reduced background traffic (see next section).

Figure 7 shows the measured RTT of the CloudMAC traffic between the $\mathrm{MN}$ and the VAP over time using different queuing strategies. As we can see, the RTT of CloudMAC traffic between WTP and VAP remains low and stable when there is no cross traffic introduced. This is similar to the traffic

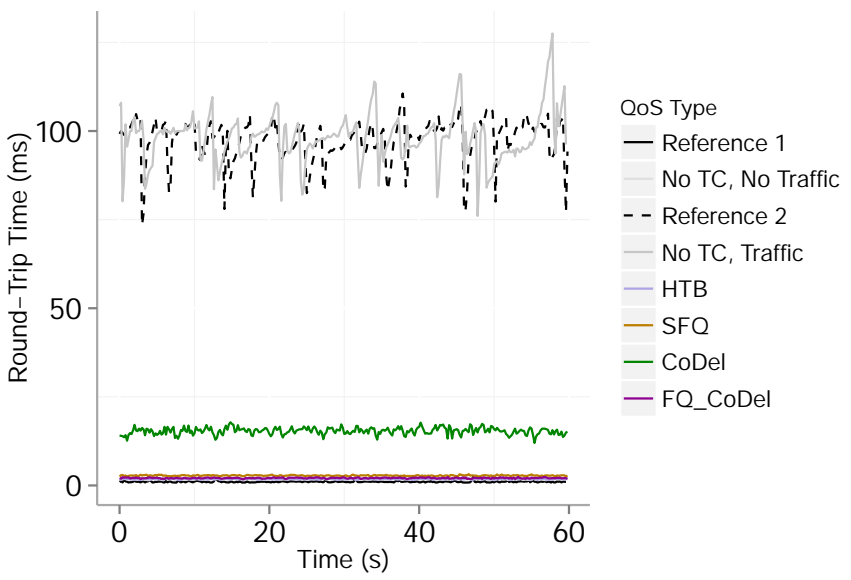

Fig. 7: RTT between $\mathrm{MN}$ and AP/VAP over time

of Reference 1 (traditional wireless network setup). When cross traffic is introduced, the RTT is higher and less stable. This is also true for the routed wireless network (Reference 2). CoDel provides a decrease in RTT and increase in stability compared to plain FIFO. It is however not so good compared to strategies such as SFQ and FQ_CoD because all traffic flows are processed in a single queue, causing an increased RTT and decreased stability. Fair queueing strategies like SFQ and FQ_CoDel and strict prioritisation strategies like HTB all provide very low RTT and high stability, because CloudMAC traffic is assigned an individual prioritised queue.

\section{Impact of cross traffic and queuing strategies on through- put}

We also measured the TCP throughput of the CloudMAC data from the MN to the VAP (Figure 8) using different queueing strategies. Each test was repeated 15 times and run for 60 seconds per test.

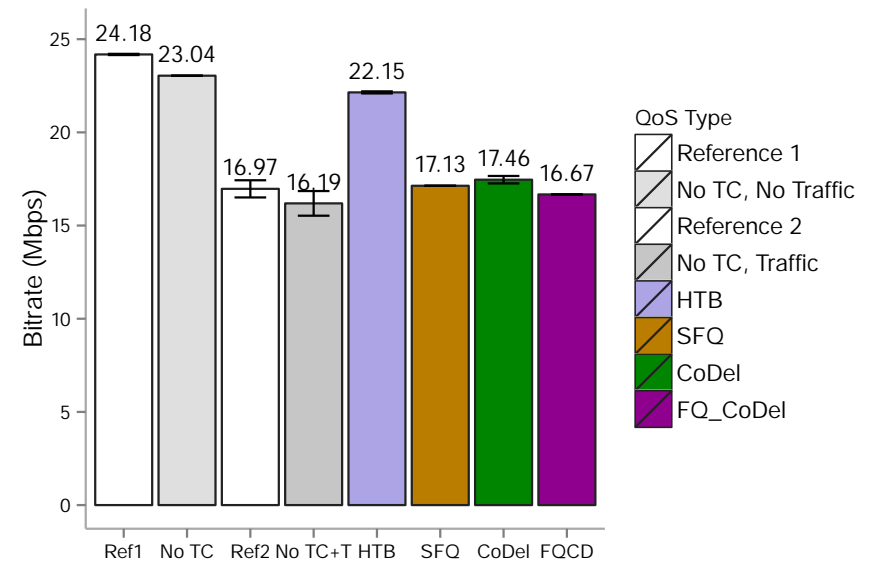

Fig. 8: TCP performance from MN to AP/VAP

In Figure 8, we can see that the measured TCP throughput of CloudMAC traffic from the MN to the VAP is $23.041 \mathrm{Mbps}$, 
when transmitted over an uncongested network. This is $1.14 \mathrm{Mbps}$ lower throughput compared to a traditional WLAN (Reference 1: 23.041 Mbps), which is caused by the additional CloudMAC header and increased RTT as CloudMAC frames are exchanged between WTP and VAP. When the link A $\leftrightarrow$ $\mathrm{B}$ is congested, we can see a reduction in TCP throughput between the MN and the VAP. The measured CloudMAC TCP throughput is now $16.19 \mathrm{Mbps}$, which is $0.78 \mathrm{Mbps}$ less than what can be achieved in the routed wireless network. The fair queueing strategies only marginally improve the throughput of CloudMAC compared to using plain FIFO queuing. HTB traffic control algorithm however, improves the TPC throughput significantly $(22.146 \mathrm{Mbps})$ due to the strict prioritisation of CloudMAC traffic that HTB provides.

Figure 9 shows the impact that CloudMAC traffic has on the cross traffic between CT1 and CT2 using the FQ_CoDel queueing strategy. We took the first run of the 15 repetitions and analyse it over time. To recall, in this scenario the MN creates a TCP stream, which is sent through the WTP over the SDN to the VAP while cross traffic is present. In the Figure, CT FQ_CoDel (Up) denotes the aggregate uplink traffic between CT2 and CT1 (CT2 $\rightarrow$ CT1), while CT FQ_CoDel (Down) denotes the aggregate downlink traffic between CT1 and CT2 (CT1 $\rightarrow$ CT2). Cross traffic is generated from the $0 \mathrm{~s}$ mark to the $120 \mathrm{~s}$ mark. CloudMAC traffic generation starts at the $30 \mathrm{~s}$ mark and ends at the $90 \mathrm{~s}$ mark.

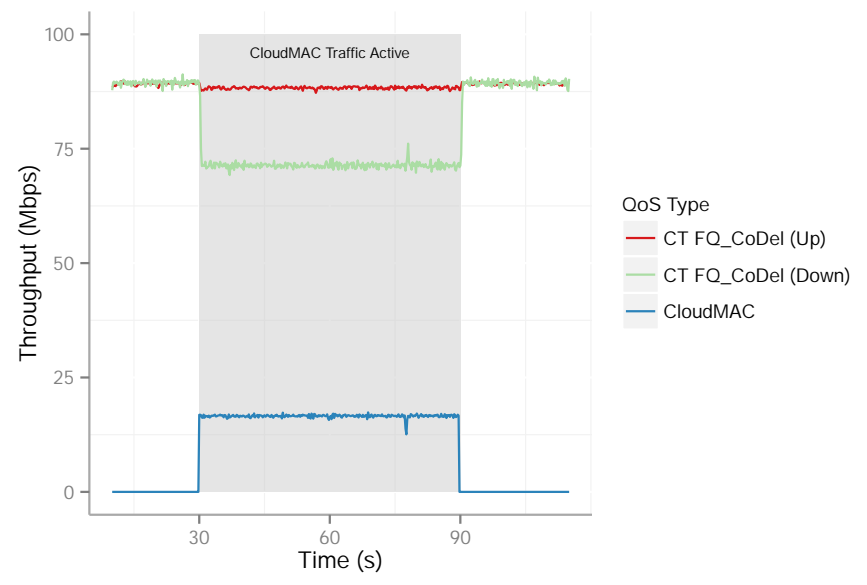

Fig. 9: CloudMAC impact on cross traffic flows using FQ_CoDel

We can see that when there is no CloudMAC traffic $(0 \mathrm{~s}-$ $30 \mathrm{~s}$ ), the cross traffic can fully utilise the throughput of the link, around (89 Mbps). When the MN starts transmitting CloudMAC traffic to the VAP $(30 s-90 s)$, the throughput of the downlink cross traffic is reduced by around $17 \mathrm{Mbps}$ to $71 \mathrm{Mbps}$. The average CloudMAC throughput between $30 \mathrm{~s}$ and $90 \mathrm{~s}$ is around $16.6 \mathrm{Mbps}$. The cross traffic uplink flow is also slightly affected, as it is reduced from $89.2 \mathrm{Mbps}$ to $88.3 \mathrm{Mbps}$. This reduction in throughput comes from the TCP ACK packets that are sent through the VAP and WTP towards the MN. Please compare the throughput also with the RTT values of FQ_CoDel which we obtained from the same run (see Figure 7, but observe that in Figure 9 we depict the whole experiment duration while in Figure 7 we zoom in to the time where CloudMAC traffic was active).

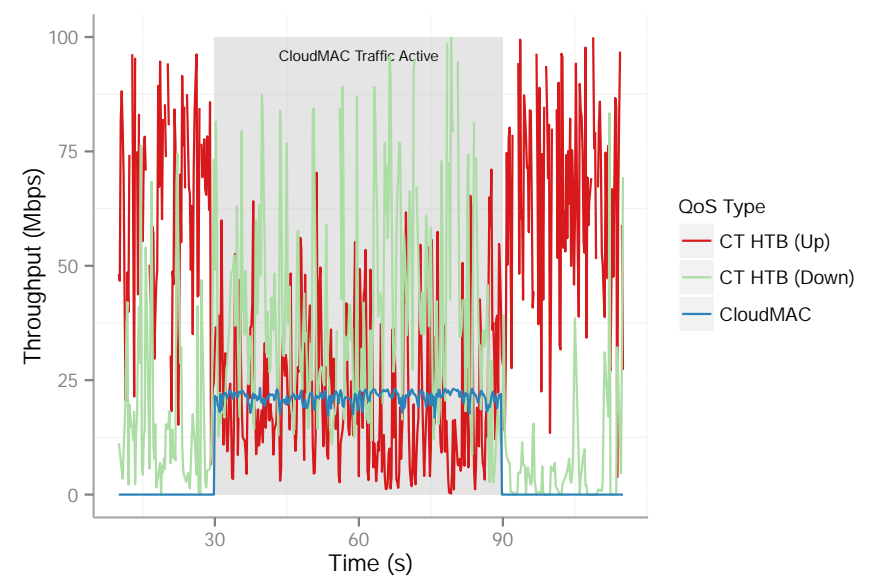

Fig. 10: CloudMAC impact on cross traffic flows using HTB

Finally, we were interested to find out the throughput performance when using HTB. We have seen already from Figure 6 and Figure 7 that HTB allows low latency CloudMAC traffic due to its strict prioritisation while at the same time achieving high throughput (HTB provides to CloudMAC around $22 \mathrm{Mbps}$ at $1.79 \mathrm{~ms}$ RTT while FQ_CoDel provides CloudMAC with $16.67 \mathrm{Mbps}$ at $2.09 \mathrm{~ms}$ RTT). Therefore, we analysed how the throughput evolves over time when using HTB when the MN sends a TCP stream to the VAP through the WTP over CloudMAC and in parallel the cross traffic generators exchange competing TCP flows over the SDN enabled network. Figure 10 show both the throughput of cross traffic and CloudMAC over time. Again, we first start cross traffic at time $0 \mathrm{~s}$ and at time $30 \mathrm{~s}$ we start a TCP stream at the MN which is sent using CloudMAC. As we can see from the Figure, CloudMAC achieves higher throughput compared to the FQ_CoDel case. But the throughput fluctuates more and is less stable. In addition, cross traffic is much more penalised with HTB in terms of throughput. For example, the uplink traffic CT HTB (up) is reduced to $21.87 \mathrm{Mbps}$ during the presence of CloudMAC traffic while the downlink traffic is reduced to $42.93 \mathrm{Mbps}$. In addition, both uplink and downlink traffic are much more unstable compared to when using FQ_CoDel.

\section{E. Evaluation Summary}

As we observed in our experiments, cross traffic significantly impacts CloudMAC traffic, increasing the RTT and lowering both the throughput for mobile stations and their connection success rate. However, utilising clever queueing strategies can successfully prevent this performance degradation. Using HTB, a strict prioritisation queueing strategy where each queue can be configured with separate rate limitations and priorities, the performance loss of CloudMAC due to cross 
traffic can be mitigated. However, this comes at the expense of unstable cross traffic along with much reduced rate. On the other hand, fair queueing strategies like FQ_CoDel and SFQ can help to provide low RTT and prevent connection issues for CloudMAC, but do somehow penalise CloudMAC throughput, because of their classless operation. This showcases the importance of proper QoS management integrated into SDN operation for NFV for WiFi MAC layer processing in the Cloud.

\section{CONClusion}

In this paper, we have taken a first step towards providing WiFi MAC layer processing as a NFV service using CloudMAC. We separate a WiFi AP into a simple physical MAC layer forwarding device and an intelligent software based AccessPoint which is virtualised and runs within a VM in a co-located private cloud such as using OpenStack. As CloudMAC traffic traverses the network, we have developed effective prioritization of CloudMAC traffic using an SDN based approach, where the SDN controller allocates priority queues for CloudMAC traffic and creates rules that prioritise it. An evaluation in our testbed showed that queueing strategies can effectively prioritise CloudMAC traffic while minimising the impact on background traffic both in terms of latency and throughput.

As a next step in our work on Network Function Virtualisation we are going to evaluate the scalability of the system with respect to VM sizing. We will evaluate the impact of different VM configurations such as number of virtual CPUs or the virtualisation drivers on CloudMAC performance. Also, we will evaluate the impact of different prioritisation strategies on CloudMAC traffic between the VAP and WTP using our QoS extensions for OpenFlow, like prioritising management frames more than normal data frames, which can be achieved by updating the matching and forwarding rules. This we will then couple with MAC layer priorisation of WiFi management frames in the air using IEEE 802.11ae [16].

\section{ACKNOWLEDGEMENTS}

Part of this work was sponsored by Knowledge Foundation (KKStiftelsen) through the project READY.

\section{REFERENCES}

[1] Nick McKeown et al. OpenFlow: Enabling Innovation in Campus Networks. Tech. rep. Mar. 2008.

[2] Haiyong Xie et al. "vRGW: Towards network function virtualization enabled by software defined networking". In: Network Protocols (ICNP), 2013 21st IEEE International Conference on. 2013, pp. 1-2. DOI: 10.1109/ ICNP.2013.6733632.

[3] OpenStack. OpenStack. URL: http://www.openstack. org/.

[4] P. Dely et al. "CloudMAC - An OpenFlow based architecture for 802.11 MAC layer processing in the cloud". In: Globecom Workshops (GC Wkshps), 2012 IEEE. Dec. 2012, pp. 186-191.
[5] S. Govindan et al. Objectives for Control and Provisioning of Wireless Access Points (CAPWAP). RFC 4564 (Informational). Internet Engineering Task Force, July 2006. URL: http://www.ietf.org/rfc/rfc4564.txt.

[6] Lalith Suresh et al. "Towards Programmable Enterprise WLANS with Odin". In: Proceedings of the First Workshop on Hot Topics in Software Defined Networks. HotSDN '12. Helsinki, Finland: ACM, 2012, pp. 115120. ISBN: $978-1-4503-1477-0$. DOI: $10.1145 / 2342441$. 2342465. URL: http://doi.acm.org/10.1145/2342441. 2342465 .

[7] A. Ishimori et al. "Control of Multiple Packet Schedulers for Improving QoS on OpenFlow/SDN Networking". In: Software Defined Networks (EWSDN), 2013 Second European Workshop on. Oct. 2013, pp. 81-86.

[8] M. Said Seddiki et al. "FlowQoS: QoS for the Rest of Us". In: Proceedings of the Third Workshop on Hot Topics in Software Defined Networking. HotSDN '14. Chicago, Illinois, USA: ACM, 2014, pp. 207-208. ISBN: 978-1-4503-2989-7. DOI: 10 . 1145 / 2620728 . 2620766. URL: http://doi.acm.org/10.1145/2620728. 2620766.

[9] J.L. Valenzuela et al. "A hierarchical token bucket algorithm to enhance QoS in IEEE 802.11: proposal, implementation and evaluation". In: Vehicular Technology Conference, 2004. VTC2004-Fall. 2004 IEEE 60th. Vol. 4. Sept. 2004, 2659-2662 Vol. 4.

[10] P.E. McKenney. "Stochastic fairness queueing". In: INFOCOM '90, Ninth Annual Joint Conference of the IEEE Computer and Communication Societies. The Multiple Facets of Integration. Proceedings, IEEE. 1990, 733-740 vol.2. DOI: 10.1109/INFCOM.1990. 91316.

[11] Kathleen Nichols and Van Jacobson. Controlling Queue Delay. A modern AQM is just one piece of the solution to bufferbloat. Tech. rep. May 2012.

[12] Sally Floyd and V. Jacobson. "Random early detection gateways for congestion avoidance". In: Networking, IEEE/ACM Transactions on 1.4 (Aug. 1993), pp. 397413. ISSN: 1063-6692. DOI: 10.1109/90.251892.

[13] T. Hoeiland-Joergensen et al. FlowQueue-Codel. Mar. 2014. URL: https://tools.ietf.org/html/draft-hoeilandjoergensen-aqm-fq-codel-00.

[14] The OpenDaylight Project. OpenDaylight. URL: http: //www.opendaylight.org/.

[15] tohojo/netperf-wrapper github. URL: https://github.com/ tohojo/netperf-wrapper.

[16] IEEE 802.11ae 2012. Standard for Information Technology - Telecommunications and Information Exchange Between Systems - Local and Metropolitan Area Networks - Specific Requirements - Part 11: Wireless LAN Medium Access Control (MAC) and Physical Layer (PHY) Specifications; Amendment: Prioritization of management frames. 